\title{
The Isoelectric Point of Lead Magnesium Niobate
}

\author{
Aylin M. Deliormanll, ${ }^{\dagger, \ddagger}$ Erdal Çelik, ${ }^{\S}$ and Mehmet Polat ${ }^{\ddagger}$ \\ ${ }^{\ddagger}$ Chemical Engineering Department, Izmir Institute of Technology, Urla, Izmir, Turkey \\ ${ }^{\S}$ Metallurgy and Materials Engineering Department, Dokuz Eylül University, Izmir, Turkey
}

\begin{abstract}
Lead magnesium niobate (PMN) is an important relaxor ferroelectric material commonly used in multilayer capacitor and actuator manufacturing owing to its high dielectric constant and superior electrostrictive properties. However, the isoelectric point of this material in water is not known and there is justification for a detailed investigation. In this work, the isoelectric point (IEP) of aqueous PMN suspensions were determined as a function of solids concentration. Results showed that IEP of the PMN suspensions strongly depended on the solids loading. The IEP was between pH 9 and 10 at particle concentrations between 10 to 20 vol\%. The IEP shifted gradually to a lower $\mathrm{pH}$ value as the particle concentration decreased. Solubility experiments showed that $\mathbf{P b}^{2+}$ and $\mathrm{Mg}^{2+}$ ions dissolved from the PMN surface, especially in the acidic $\mathrm{pH}$ range. The study provides a new insight on the aqueous stability of perovskite materials which possess more than one soluble cation in their structure.
\end{abstract}

\section{Introduction}

$\mathrm{L}$ EAD magnesium niobate (PMN) is a relaxor ferroelectric material, which is characterized by a diffuse phase transition over a broad temperature range and a frequency-dependent maximum in its relative dielectric permittivity. ${ }^{1}$ It has many potential applications such as multilayer ceramic capacitors, actuators, and electro-optic devices. ${ }^{2}$ It also finds applications in motors, pumps, optical scanning systems, and vibration isolators. ${ }^{3}$

The manufacturing of multilayer electronic devices composed of perovskite materials such as PMN generally involves a colloidal process, which requires preparation of colloidal suspensions. However, the behavior of PMN particles in water medium has yet to be clarified. The few studies on this subject include the preparation of PMN-lead titanate (PMN-PT) suspensions in solvents other than water ${ }^{4-6}$ whereas detailed work is present on barium titanate system in water. ${ }^{7,8}$ Therefore, understanding of the $\mathrm{PMN}-\mathrm{H}_{2} \mathrm{O}$ system is essential in producing high-quality PMN ceramics.

The isoelectric point (IEP) is an important parameter in the $\mathrm{PMN}-\mathrm{H}_{2} \mathrm{O}$ system because it determines the stability of the colloidal particles in liquid medium. It is the $\mathrm{pH}$ value at which the apparent charge of the particles is zero. ${ }^{9}$ Therefore, the electrostatic repulsion between the interacting particles is eliminated at the IEP. Under such a condition, particles become unstable and tend to coagulate. Ceramic oxides generally have well defined and characteristic IEP values. ${ }^{10} \mathrm{On}$ the other hand, it is possible for a powder to exhibit multiple IEP values due to specific adsorption, dissolution, and/or precipitation reactions. ${ }^{11,12}$

V. Hackley—contributing editor

Manuscript No. 23051. Received April 7, 2007; approved May 19, 2007

The work has been funded by the Scientific and Research Council of Turkey (TUBITAK), Grant, NATO-A2. This work is financially supported by the State Planning Organization of Turkey.

Author to whom correspondence should be addressed. e-mail: aylindeliormanli@ iyte.edu.tr
Perovskite materials such as $\mathrm{PMN}$ have the general chemical formula, $\mathrm{ABO}_{3}(\mathrm{~A}=\mathrm{K}, \mathrm{Na}, \mathrm{Ca}, \mathrm{Ba}, \mathrm{Pb}, \mathrm{Mg}$, etc. $)$ and $(\mathrm{B}=\mathrm{Ti}$, $\mathrm{Nb}, \mathrm{Zr}$, etc.). In perovkites, the cation at the A site is generally soluble in water whereas the B-site cation that is only slightly soluble overall the practical $\mathrm{pH}$ range. Therefore, the A-site cation undergoes incongruent dissolution leaving an interface that is relatively rich in the B-site cation. Because of this fact, determination of the IEP of the perovskite materials such as PMN cannot be made by titration of the stock suspensions at a single solids loading. ${ }^{13}$

Paik and Hackley investigated the effect of solids concentration on the IEP of barium titanate. They reported that the particle surface charge was influenced by solids concentration in aqueous suspensions of barium titanate. They observed a systematic acidic shift in the isoelectric $\mathrm{pH}$ with decreasing solids concentration. ${ }^{7}$

In this study, the dependence of the IEP of PMN suspensions on solution $\mathrm{pH}$ and solids loading was investigated. The solubility characteristics of the cations that constitute PMN were examined as a function of $\mathrm{pH}$.

\section{Experimental Procedure}

$\mathrm{PMN}, \mathrm{Pb}\left(\mathrm{Mg}_{1 / 3} \mathrm{Nb}_{2 / 3}\right) \mathrm{O}_{3}$ powder produced by the combustion spray pyrolysis method was provided by Praxair Specialty Ceramics (Woodinville, WA). Powder purity is $99.9 \%$ as reported by the manufacturer. Bulk density (Helium pycnometer Micromeritics 1330) and the Brunauer-Emmett-Teller surface area (Micromeritics ASAP 2400, Norcross, GA) of the powder are measured to be $7.967 \mathrm{~g} / \mathrm{cm}^{3}$ and $1.168 \mathrm{~m}^{2} / \mathrm{g}$, respectively. Particle size distribution of the powder was measured using a particle size analyzer (Model CAPA-700, Horiba, Ltd., Tokyo, Japan) and the $d_{50}$ of the powder was around $2.0 \mu \mathrm{m}$. Other materials used in the work are Niobium (V) oxide (Alfa Aesar, 99.9\% metal basis), lead (II) oxide (Alfa Aesar, Ward Hill, MA, 99.9\%), and magnesium oxide (Analytical reagent, Mallinckrodt AR, Paris).

Aqueous PMN suspensions of varying $\mathrm{pH}$ values were prepared at different solids loadings ranging from 0.001 to $20 \mathrm{vol} \%$ using deionized water. Suspensions were magnetically stirred for $24 \mathrm{~h}$ prior the measurements at room temperature. $\mathrm{pH}$ adjustments were performed using $1 M$ solutions of $\mathrm{HNO}_{3}$ and $\mathrm{NH}_{4} \mathrm{OH}$. The $\mathrm{pH}$ of the suspensions were controlled during the stirring period and further adjustment were performed when necessary. Because the addition of simple electrolytes do not affect the IEP (although it affects the magnitude of the $\zeta$ potential), no care was taken to control the electrolyte concentration such as by using a background electrolyte.

Solubility of $\mathrm{Pb}^{2+}, \mathrm{Mg}^{2+}$, and $\mathrm{Nb}^{5+}$ ions in the PMN structure was examined using inductively coupled plasma-atomic emission spectrometer (ICP-AES Optima 2000 DV Perkin Elmer, Norwalk, CT). For this purpose, suspensions prepared at 5 $\mathrm{vol} \%$ according to the procedure described in the previous section were centrifuged at $4000 \mathrm{rpm}$ for $1 \mathrm{~h}$ to obtain a clear supernatant. Supernatants were further filtered from $0.02 \mu \mathrm{m}$ 
microfilters to eliminate any possible particles in solution. The amounts of $\mathrm{Pb}^{2+}, \mathrm{Mg}^{2+}$, and $\mathrm{Nb}^{5+}$ in particle-free supernatants were determined using ICP-AES at different $\mathrm{pH}$ values.

$\zeta$ potential measurements were performed using a $\zeta$ sizer (Malvern 3000 HS, Malvern Instruments, Ltd., Worcestershire, U.K.) with aqueous dip cell apparatus whose electrodes were properly cleaned before each measurement to avoid contamination. The device makes use of the laser Doppler microelectrophoresis technique for the $\zeta$ potential determination. The $\zeta$ potential of particles was calculated from their electrophoretic mobility by means of the Henry correction of the Smoluchowski equation ${ }^{14}$ :

$$
\mu=\frac{2 \varepsilon \zeta}{3 \eta} f(\kappa a)
$$

where $\mu$ is the electrophoretic mobility, $\eta$ is the aqueous solution viscosity, and $f(\kappa a)$ the Henry coefficient. In this work an $f(\kappa a)$ of 1.5 was used through the measurements. Because this technique allows measurements only in dilute suspensions, samples were diluted before measurement using sedimentaion and followed by redispersing of a small amount of sediment redispersed with the supernatant. The measurements were periodically checked against a calibration standard with a $\zeta$ potential of $-50( \pm 5)$ $\mathrm{mV}$. Experiments were performed in triplicate, and the results given are the average of 10 measurements.

$\zeta$ potentials of concentrated suspensions were also determined directly by electrokinetic sonic amplitude measurements using an electroacoustic analyzer (ESA-9800, Matec Applied Sciences, Northborough, MA). The solids loadings in this case were $0.1,5$, and $10 \mathrm{vol} \%$. The ESA apparatus makes use of the so-called electroacoustic effect created by the application of a high-frequency $\mathrm{AC}$ field in the order of $1 \mathrm{MHz}$ to the suspension. If particles carry any electrostatic charge, they will oscillate around the position of rest. Such an oscillation generates a pressure wave in the liquid that can be detected and correlated to the $\zeta$ potential. Because the technique is capable of measuring the $\zeta$ potential of concentrated suspensions, the samples were used without any dilution. To ensure dispersion the suspensions were ultrasonicated for $2.5 \mathrm{~min}$ using an ultrasonic horn (550 Sonic Dismembrator, Fisher Scientific, Pardubice, Czech Republic) before the measurements. The ESA measurements were checked against a $10 \mathrm{vol} \%$ Ludox suspension having a $\zeta$ potential of -38 $\mathrm{mV}$. Experiments were performed in triplicate, and the results given are the average of 10 measurements.

\section{Results and Discussion}

Cation dissolution from the PMN surfaces was examined as a function of solution $\mathrm{pH}$. Figure 1 indicates the amount of cation concentration in solution after $24 \mathrm{~h}$ for a solids loading of 5

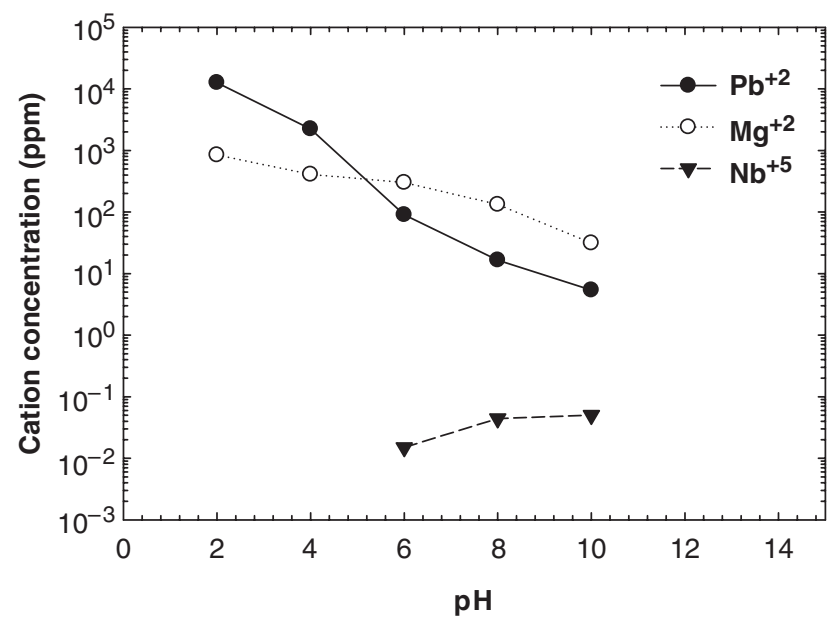

Fig. 1. Dissolved $\mathrm{Pb}^{2+}, \mathrm{Mg}^{2+}$, and $\mathrm{Nb}^{+5}$ ion concentration as a function of solution $\mathrm{pH}$, solids loading $5 \mathrm{vol} \%$. vol\%. The graph shows that the lead and magnesium ion concentrations increase with decreasing $\mathrm{pH}$. Especially the $\mathrm{Pb}^{2+}$ concentration increases dramatically in the acidic region and reaches $12620 \mathrm{ppm}$ at $\mathrm{pH} 2$. Though the $\mathrm{Mg}^{2+}$ dissolution was also $\mathrm{pH}$ dependent, the dissolution rate was almost an order of magnitude smaller compared with that observed with the $\mathrm{Pb}^{+2}$ at low $\mathrm{pH}$ values. But it was observed that $\mathrm{Mg}^{2+}$ dissolution was significant at neutral $\mathrm{pH}$ values. On the other hand, $\mathrm{Nb}^{+5}$ showed a rather low dissolution under the same conditions. The $\mathrm{Nb}^{+5}$ concentrations in the acidic region were below the detection limit of the analyzing device. Therefore, PMN behaves both like an insoluble oxide and like a partially soluble inorganic salt. Insoluble oxide can be approximated to the $\mathrm{Nb}_{2} \mathrm{O}_{5}$ component whereas the salt is in the form of $\mathrm{PbO}$ and $\mathrm{MgO}$ in the PMN structure.

Previously, Neubrand et al. ${ }^{8}$ investigated the dissolution of $\mathrm{Ba}^{2+}$ ions from the barium titanate in water. They found that the amount of barium leached increased as the $\mathrm{pH}$ decreases. In fact, the increase in the dissolved metal ion concentration with decreasing $\mathrm{pH}$ was considered to be a general behavior for most perovskites in water.

Adair et al. ${ }^{13}$ reported that the dissolution types were dictated by the ceramic materials and the solution $\mathrm{pH}$. According to their study most multicomponent metal oxides such as barium titanate generally follow incongruent types of dissolution, but the dissolution mechanism strongly depends on the $\mathrm{pH}$. In our study, similar to barium titanate system in the absence of any surface carbonates, the $\mathrm{Pb}-\mathrm{Mg}-\mathrm{Nb}-\mathrm{H}_{2} \mathrm{O}$ system undergoes incongruent dissolution with selective leaching of $\mathrm{Pb}^{2+}$ and $\mathrm{Mg}^{2+}$ in the $\mathrm{pH}$ range between $\mathrm{pH} 2$ and 10. However, unlike the barium titanate, in the PMN system niobium oxide-rich matrix does not totally dissolve below $\mathrm{pH} 2$. Because the solubility of $\mathrm{Nb}^{5+}$ was very low compared with other cations in PMN structure and increases with increasing $\mathrm{pH}$ as shown by the experiments. Previously Paik and Hackley ${ }^{7}$ showed that for barium titanate system, the dissolution process can be divided into three stages. First stage is an incongruent dissolution in the first few minutes after initial contact with the acidic solution. It is followed by a mass transport-limited second step. A similar dissolution process is valid for the PMN-water system. In the first step, $\mathrm{Pb}^{2+}$ and $\mathrm{Mg}^{2+}$ must diffuse out through a growing reactive surface layer. In the final stage, dissolution slows down considerably and the following possibilities may arise: a steadystate equilibrium concentration is reached, a thick the depletion layer may form and leads to passivation; or the particles are completely converted to Nb-rich phase. ${ }^{7}$

Figures 2 and 3 show the variation in $\zeta$ potential as a function of solids concentration obtained by microelectrophoresis and electroacoustic methods, respectively. Although slight differences were observed, in general the IEP values obtained using both methods were in good agreement with each other. As it was

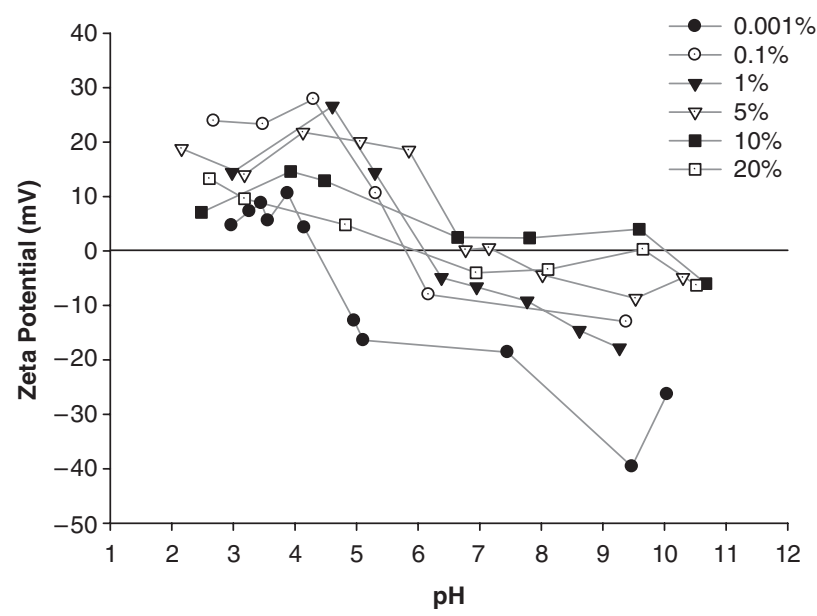

Fig. 2. The isoelectric point of lead magnesium niobate suspensions at various solids loadings, microelectrophoresis method. 


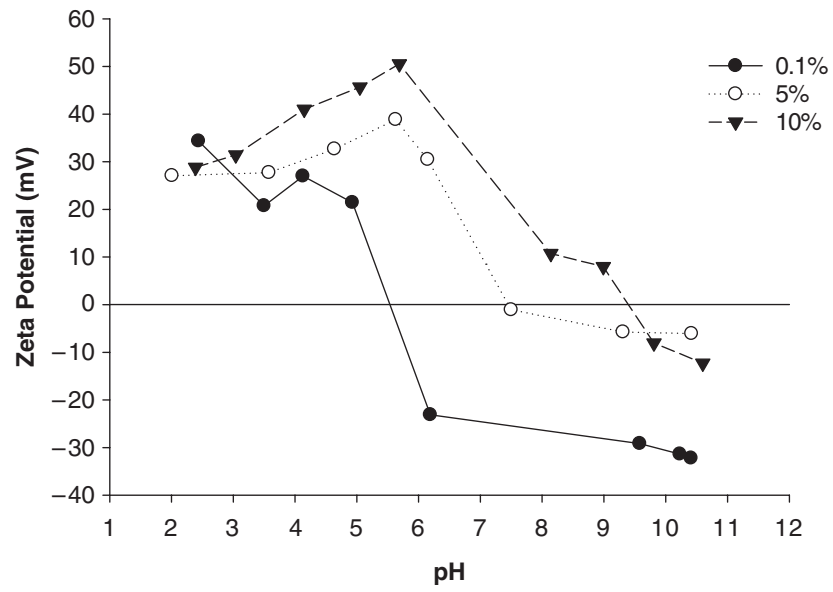

Fig. 3. The isoelectric point of lead magnesium niobate suspensions at various solids loadings, electroacoustic method.

described in the previous section, the electrophoresis method requires the use of extremely dilute suspensions. However, the $\zeta$ potential of concentrated suspensions can be directly measured using the electroacoustic method. In the current study samples for the electrophoresis technique were prepared from concentrated suspension by dilution. It is also important to note that the IEP values should depend only on the solid-liquid interfacial chemistry and not on the technique used to measure them.

IEP values that were determined using both the techniques were also tabulated in Table I. The agreement between microelectrophoresis and electrokinetic sonic amplitude techniques at the overlap solids concentration of $0.1 \%$ serves to validate the methodology.

The shift in the IEP of the PMN suspensions to lower $\mathrm{pH}$ values at low solids concentrations can be explained based on the solubility considerations. When the PMN is suspended in water a surface layer rich in residual $\mathrm{Nb}^{+5}$ ions will be developed around the particle due to the deficiency of the dissolving $\mathrm{Pb}^{2+}$ and $\mathrm{Mg}^{2+}$ ions. Specifically, the surface layer forms because of mass-transport limited incongruent dissolution. Without the mass-transport limitation, the entire particle would be converted to the $\mathrm{Nb}$ oxide at sufficiently low $\mathrm{pH}$ and low solids concentration. The thickness of that layer is determined by both the $\mathrm{pH}$ to which it is subjected and the surface-to-volume ratio of the solid phase. A lower $\mathrm{pH}$ increases the dissolution rate and also accommodates a higher solubility limit for the dissolving Atype cations. Essentially, an $\mathrm{Nb}$ oxide-rich corona develops around a core of unmodified perovskite material. The thickness and level of depletion of the A-type cations in this corona determines the "acidity" of the particles. That is, the greater the depletion and the thicker the depletion layer, the lower the IEP, until the IEP approaches that of the more acidic $\mathrm{Nb}$ oxide. The solids concentration effect is due to the fact that at higher solids, more particle surface is available for dissolution to occur. This

Table I. IEP of PMN Suspensions at Different Solids Concentrations

\begin{tabular}{lcc}
\hline & \multicolumn{2}{c}{ IEP } \\
\cline { 2 - 3 } $\begin{array}{l}\text { Solids concentration } \\
\text { (vol\%) }\end{array}$ & Microelectrophoresis & $\begin{array}{c}\text { Electrokinetic sonic } \\
\text { amplitude (ESA) }\end{array}$ \\
\hline 0.001 & 4.4 & - \\
0.1 & 5.8 & 5.6 \\
1 & 6.1 & - \\
5 & 7.1 & 7.5 \\
10 & 10 & 9.6 \\
20 & 9.7 & - \\
\hline
\end{tabular}

IEP, isoelectric point; ESA, electroacoustic analyser.

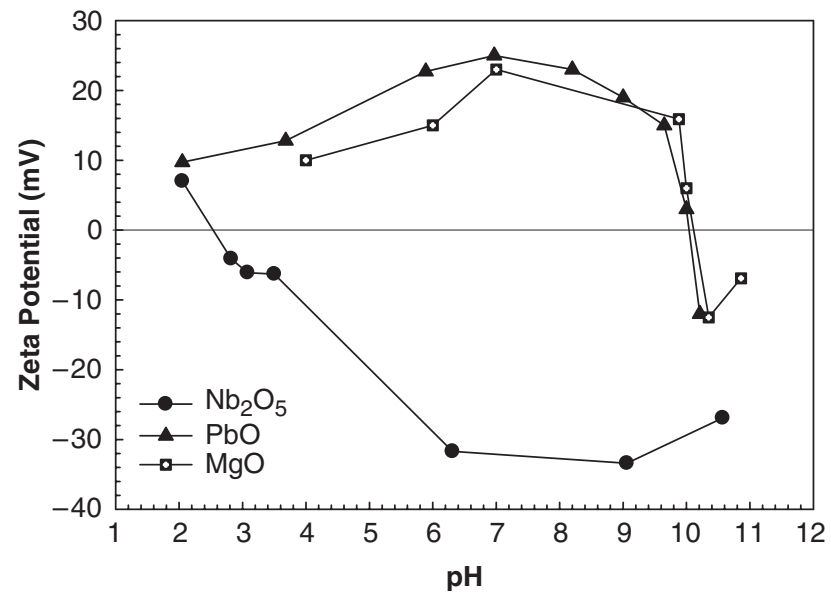

Fig. 4. Isoelectric point of individual metal-oxides which constitute the lead magnesium niobate. Solids loading: $10^{-2}$ vol $\%$.

means that the $\mathrm{pH}$-determined solubility limit of the dissolving cations will be reached more quickly and with less depletion of cations from any given particle. Thus the apparent IEP will not shift as much from the native material. At lower solids, the depletion zone of all particles in the suspension must increase in order to reach the same solubility limit for a given $\mathrm{pH}$ since the surface-to-volume ratio within the suspension is reduced. This results in a greater (acidic) shift in the IEP. ${ }^{7}$

The IEP of oxides that constitute the PMN were measured and the results are shown in Fig. 4. In the current study the IEP of native niobium oxide is determined at $\mathrm{pH}$ 2.6. Previously, Kosmulski ${ }^{15}$ has reported the IEP of niobium oxide at $\mathrm{pH} 4.5$. Therefore, the shift in the IEP of PMN suspensions to lower $\mathrm{pH}$ values may also be explained by the formation of a $\mathrm{Nb}_{2} \mathrm{O}_{5}$-rich structure at low solids concentrations.

\section{Conclusions}

Chemical stability of PMN powders in water was investigated in this paper. Solubility experiments showed that $\mathrm{Pb}^{2+}$ and $\mathrm{Mg}^{2+}$ ions preferentially dissolve from the PMN surface as a function of $\mathrm{pH}$. Dissolution of $\mathrm{Pb}^{2+}$ and $\mathrm{Mg}^{2+}$ increases with decreasing $\mathrm{pH}$ while $\mathrm{Nb}^{5+}$ dissolution is negligible under the same conditions. It is concluded that such incongruent dissolution in the $\mathrm{Pb}-\mathrm{Mg}-\mathrm{Nb}-\mathrm{H}_{2} \mathrm{O}$ system takes place in a wide $\mathrm{pH}$ range between $\mathrm{pH} 2$ and 10.

IEP of PMN suspensions ( $10 \mathrm{vol} \%)$ in water was measured to be around $\mathrm{pH} 9.5$ and $\mathrm{pH} 10$ using electroacoustic and electrophoresis techniques, respectively. It was found that IEP shifts to lower $\mathrm{pH}$ values with decreasing solids concentration. The positive surface charge of the PMN was attributed to protonation of the amphoteric oxide groups and also to dissolution and readsorption of the dissolved cations from the solution phase to the PMN surface.

\section{Acknowledgments}

$\zeta$ potential and ICP-AES measurements were performed at University of Illinois at Urbana Champaign, U.S.A. Invaluable advice and support of Prof. Dr. Jennifer A. Lewis is gratefully acknowledged.

\section{References}

${ }^{1}$ S. Fengbing, L. Qiang, Z. Haisheng, L. Chunhong, Z. Shix, and S. Dezhong, "Phase Formation and Transition in the Lead Magnesium Niobate-Lead Titanate," Mater. Chem. Phys., 83, 135-139 (2004).

${ }^{2}$ A. Brailenau, A. Ianculescu, M. Zaharescu, I. Pasuk, S. Preda, J. Madarasz, and G. Pokol, "Phase Formation Study of La-Modified Lead Magnesium Niobate," Key Eng. Mater., 1309, 264-8 (2004).

${ }^{3}$ H. S. Tzou, H. J. Lee, and S. M. Arnold, "Smart Materials, Precision Sensors Actuators, Smart Structures, and Structronic Systems," Mech. Adv. Mater. Struct., 11, 367-93 (2004) 
${ }^{4}$ H. Windlass, P. Markondeya Raj, and D. Balamaran, "Colloidal Processing of Polymer Ceramic Nanocomposites for Integral Capacitors," pp. 393-8, International Symposium on Advanced Packaging Materials: Processes, Properties and Interfaces, Proceedings. Braselton, Georgia, USA (2001).

${ }^{5}$ S. Ogitani, S. Bidstrup-Allen, and P. Kohl, "Development of High-K Photo Definable Epoxy Composites for Embedded Capacitors," pp. 199-205, IEEE/ CPMT International Electronics Manufacturing Technology Symposium Proceedings. Austin, TX (1998).

${ }^{6}$ V. Agarval, P. Chahal, R. R. Tummala, and M. G. Allen, "Improvements and Recent Advances in Nano Composite Capacitors Using a Colloidal Technique," pp. 165-70, IEEE, Electronic Components and Technology Conference Proceedings, Seattle, WA (1998)

${ }^{7}$ U. Paik and V. A. Hackley, "Influence of Solids Concentration on the Isoelectric Point of Aqueous Barium Titanate," J. Am. Ceram. Soc., 83, 2381-4 (2000).

${ }^{8}$ A. Neubrand, R. Lindner, and P. Hoffmann, "Room Temperature Solubility Behaviour of Barium Titanate in Aqueous Media," J. Am. Ceram. Soc., 83 [10] $860-4(2000)$
${ }^{9} \mathrm{~W}$. Stumm, Chemistry of the Solid-Water Interface, Processes at the MineralWater and Particle-Water Interface in Natural Systems. John Wiley and Sons Inc. 1992.

${ }^{10}$ B. M. C. Lopez, B. Rand, and F. L. Riley, "The Isoelectric Point of $\mathrm{BaTiO}_{3}$," J. Eur. Ceram Soc., 20, 108-18 (2000).

${ }^{11}$ U. Paik, S. Lee, and V. A. Hackley, "Influence of Barium Dissolution on the Electrokinetic Properties of Colloidal $\mathrm{BaTiO}_{3}$ in an Aqueous Medium," J. Am. Ceram. Soc., 86 [10] 1662-8 (2003).

${ }^{12}$ U. Paik, J.-G. Yeo, M.-H. Lee, V. A. Hackley, and Y.-G. Jung, "Dissolution and Precipitation of Barium at the Particulate $\mathrm{BaTiO}_{3}$ Aqueous Solution Interface," Mater. Res. Bull., 37, 1623-31 (2002).

${ }^{13}$ J. H. Adair, J. Crampo, M. M. Mandanas, and E. Suvaci, "The Role of Material Chemistry in Processing $\mathrm{BaTiO}_{3}$ in Aqueous Suspensions," J. Am. Ceram. Soc., 89, 1853-60 (2006).

${ }^{14}$ Malvern Instruments UK, manual no MAN0150, [2.0], 2000.

${ }^{15}$ M. Kosmulski, "Attempt to Determine Pristine Points of Zero Charge of $\mathrm{Nb}_{2} \mathrm{O}_{5}, \mathrm{Ta}_{2} \mathrm{O}_{5}$, and $\mathrm{HfO}_{2}$," Langmuir, 13 [23] 6315-20 (1997). 